\title{
Thermostatistics with an invariant infrared cutoff
}

\author{
M. Roushan ${ }^{\mathrm{a}}$, K. Nozari ${ }^{\mathrm{b}}$ \\ Department of Theoretical Physics, Faculty of Science, University of Mazandaran, Babolsar P. O. Box 47416-95447, Iran
}

Received: 21 April 2020 / Accepted: 19 August 2020 / Published online: 9 September 2020

(C) The Author(s) 2020

\begin{abstract}
Quantum gravitational effects may affect the large scale dynamics of the universe. Phenomenologically, quantum gravitational effect at large distances can be encoded in an extended uncertainty principle that admits a minimal measurable momentum/energy or a maximal length. This maximal length can be considered as the size of the cosmological horizon today. In this paper we study thermostatistics of an expanding universe as a gaseous system and in the presence of an invariant infrared cutoff. We also compare the thermostatistics of different eras of the evolution of the universe in two classes, Fermions and Bosons.
\end{abstract}

\section{Introduction}

Usually it is believed that quantum gravitational effects can be observed at small length scale or equivalently high energy regime [1-6]. However, there are some evidences against this perception. In fact, in recent years some efforts are devoted to explore the role of quantum gravity at cosmological scales and specially on the late time cosmological dynamics. In Ref. [7] the authors explored the asymptotic regimes of quantum gravity at large distances. The idea of revealing quantum gravitational effects at large distances via extended uncertainty principle was firstly reported in [8]. Page in Ref. [9] has argued the existence of huge quantum gravity effects in the Solar System. Late time cosmological dynamics with an infrared cutoff is treated in different perspectives recently in Refs. [10]. Very recently, Anagnostopoulos et al. via a paper (that has achieved honorable mention award in gravity research foundation awards) have argued that IR quantum gravity solves naturally cosmic acceleration and the coincidence problem [11]. Their novel idea was that the accelerated expansion of the universe can be ascribed to infrared quantum gravity modifications at astrophysical scales.

\footnotetext{
${ }^{a}$ e-mail: m.roushan@stu.umz.ac.ir (corresponding author)

b e-mail: knozari@umz.ac.ir
}

In Ref. [12] the authors studied the modification in thermodynamic properties of ideal gases and photon gas in high energy limit. Such modifications are authoritative at short distances. Black hole thermodynamics has been another attractive scope of current researches, where modification of thermodynamic properties of Schwarzschild and ReissnerNordström has been investigated in this framework [13]. Now, we will exhibit that, If quantum gravitational effects are important at large distances, where it seems to be actually the case, then thermodynamics of a gaseous system in this low energy limit is important to be studied. This is, along with the thermodynamic quantities considerations, the main motivation of the present study. For this purpose, we apply the distribution function strategy and accordingly encounter two classes of particles, Fermionic and Bosonic systems of particles. Pursuant to condensation phenomenon, an unlimited number of bosons can condense into the equal energy state, so at low temperatures, they can act very disparately than fermions.

In this process, we use the feature of the distribution function dependence on energy. So that, we substitute modified energy relation arising from the minimal momentum cutoff in the distribution function relation. In this regard, we consider the problem in the context of an extended uncertainty principle that admits naturally a minimal uncertainty in momentum measurement. This minimal momentum uncertainty nontrivially gives a minimal measurable momentum. We take the extended uncertainty principle $[X, P]=i \hbar\left(1+\eta x^{2}\right)$ the basis of our calculations to study thermodynamics of a gaseous system. Since we are working in a very low energy limit, the notion of a gaseous system for the universe ingredient is indeed sensible. It should be noted that we have done all this process once for massless particles and once again assuming that the particles are massive form. We also demonstrate that, there is good agreement between observational data and the corresponding calculated value in the presence of IR cutoff in massless case. 
The cosmological background of this scenario is interesting since the modified version of the standard quantum mechanics at low energy can be considered as a theoretical basis for cosmological models of the late time universe and its various aspects can be examined at different temperatures of the universe (which is equivalent to different periods of the evolution of the universe). In view of the fact that we are in an expanding universe, this expansion will reduce the temperature of the universe. The universe can be described as a gravitational structure that is in many aspects also a thermodynamic system. Indeed, it is a closed system, it also involves energy and entropy that operates as a system in which main components can be organized in different methods. On the other hand, thermodynamic parameters are a function of temperature and are thus affected by the expansion of the universe. In this paper, we represent that the effects of quantum gravity as a minimal momentum in IR cutoff framework also affect the late time evolution of these parameters. In this respect, we explore parameters as for instance number density, energy density, pressure, enthalpy, specific heat capacity and entropy and we analyze them numerically. The paper is structured as follows: in Sect. 2 we introduce minimal momentum (infrared trace of quantum gravitational effect) as a natural cutoff in the spacetime structure and we define phase space operators. In Sect. 3 we express that the infrared universe thermodynamics can be explained in a thermal equilibrium at the late time and we establish thermodynamics of the universe in regard to a modified dispersion relations (MDR) that recognizes a minimal momentum. In Sect. 4 we focus on the thermodynamical quantities for both Fermions and Bosons in attending an IR cutoff encoded in EUP. We determine the demanded quantities for both massless and massive particles. Specifically, we obtain modified number Density, energy Density, pressure, enthalpy, specific heat capacity and entropy in this section. The paper closes in Sect. 5 with summary and conclusion.

\section{Minimal momentum and maximal length}

In a seminal work, Hinrichsen and Kempf propound the existence of a minimal measurable momentum based on symmetry arguments [14]. Then Mignemi provided a framework for an extended uncertainty principle by modification of the standard uncertainty principle through a term proportional to the cosmological constant, leading to an infra-red cutoff as a minimal measurable momentum [15]. In this case the standard momentum space representation is no longer applicable due to the existence of the minimal measurable momentum. So, one has to work in the position space where there is no minimal measurable length. The minimal momentum in the spacetime structure can be explained by the curvature of spacetime. With these points in mind, one can define

$$
\begin{aligned}
& X^{i}=x^{i}, \\
& P^{i}=p^{i}\left(1+\eta x^{2}\right),
\end{aligned}
$$

where $\eta$ (carries units of inverse of length) is a small parameter encoding infra-red trace of quantum gravitational effect and $x^{i}$ and $p^{i}$ are components belonging to the ordinary quantum mechanics algebra $\left[x^{i}, p^{j}\right]=i \hbar \delta^{i j}$. So, the modified energy relation is deduced for this case as follows [16]

$$
\begin{aligned}
E(\vec{P}) & =\sqrt{m^{2} c^{4}+P^{2} c^{2}} \\
& =\sqrt{m^{2} c^{4}+p^{2} c^{2}\left(1-2 \eta x^{2}\right)} .
\end{aligned}
$$

It should be noted that in these spacetime structure $x^{i}$ and $p^{i}$ are phase space variables that fulfill the algebra of the commutative canonical phase space as

$\left\{x_{i}, p_{j}\right\}=-g_{i j}, \quad\left\{x_{i}, x_{j}\right\}=\left\{p_{i}, p_{j}\right\}=0$,

where $g_{i j}$ is the metric.

\section{Thermostatistics with an IR-deformed dispersion relation}

Thermal equilibrium is a type of equilibrium which is caused by the chemical equilibrium (chemical reactions amongst particles in equilibrium) and kinetic equilibrium (effective energy and momentum exchange of particles in equilibrium) simultaneously. It is reasonable to suppose that the infrared universe thermodynamics can be described in a thermal equilibrium at the late time. This is indeed the case since we are faced with a thermodynamic system with very low density of particles so that a control volume can be considered thermodynamically in equilibrium. So, it is potentially interesting to see the role of an invariant infrared cutoff, a minimal momentum, in the equilibrium thermodynamics of the late time universe. In this section we formulate thermodynamics of universe based on a modified dispersion relations (MDR) that admits a minimal momentum as described by the relation (2). In this regard, we assume the late time universe to be a combination of different particles (species) with various degrees of freedom. Density of states in the phase space in terms of internal degrees of freedom " $g$ " (statistical weight) is $\frac{g}{h^{3}}$ (by setting $\hbar=1$, density of states gets $\left.\frac{g}{(2 \pi)^{3}}\right)$. This feature is correct for both relativistic and non-relativistic regimes. Since the density of states in the phase space is independent of volume $V$, we can use it for the entire universe. By ignoring the interaction energies between the particles at the late time universe, a test particle energy is given by the relation (2) which is indeed an IR deformed dispersion relation. In the thermodynamic equilibrium, calculation of the distribution function is a basic concept which determines thermodynam- 
ics properties. It is an important result in statistical mechanics that the number of microstates or the number density can be obtained by the volume of the phase space. This number density is given by a distribution function (in standard model) as

$f(\vec{p})=\frac{1}{e^{\frac{(E-\mu)}{T}} \pm 1}$,

where + and - signs are respectively for Fermi-Dirac and Bose-Einstein statistics. We acquire the particle density $\frac{g}{(2 \pi)^{3}} f(\vec{p})$ by integrating over the momentum space. Then the following definitions (for $\eta=0$ ) are in order

$$
\begin{aligned}
n_{i} & =\frac{g_{i}}{(2 \pi)^{3}} \int f_{i}(\vec{p}) d^{3} p, \\
\rho_{i} & =\frac{g_{i}}{(2 \pi)^{3}} \int E_{i}(\vec{p}) f_{i}(\vec{p}) d^{3} p, \\
p_{i} & =\frac{g_{i}}{(2 \pi)^{3}} \int \frac{|\vec{p}|^{2}}{3 E_{i}} f_{i}(\vec{p}) d^{3} p,
\end{aligned}
$$

for each spices $i$ with mass $m_{i}$. We note that each particle species with $m_{i}, \mu_{i}, T_{i}$ has its own specific distribution function but given that we are working at late time universe, then we have $m \gg T$ with negligible $\mu_{i}$. We set $\hbar, c$ and $k_{B}$ to unity in all of our forthcoming calculations for the sake of simplicity.

In the next section, we use modified dispersion relation (2) and redefine relationships (5)-(7).

\section{Equilibrium thermodynamics with minimal momentum}

In this part, we study thermodynamics of the late time universe in the presence of quantum gravitational effects through an extended uncertainty Principle (EUP) that realizes a minimal measurable momentum. Since late time universe is essentially an infrared system, the assumption of equilibrium is reliable. We concentrate on the thermodynamical quantities for both Fermions and Bosons in the presence of an IR cutoff encoded in EUP as $\Delta X \Delta P \geq \frac{\hbar}{2}\left(1+\eta(\Delta X)^{2}\right)$. We calculate the required quantities for both massless and massive particles.

\subsection{Number density, energy density and pressure}

To obtain the number density in the phase space as a main quantity in thermodynamical studies, we put relations (2) and (4) into Eq. (5) and apply some approximations to get the number density of massless particles in the presence of a minimal measurable momentum as follows

$$
\begin{aligned}
& n_{\text {eff }}=\frac{4 \pi g}{(2 \pi)^{3}} \int_{P_{\min }}^{\infty} \frac{p^{2}}{e^{\frac{p c\left(1-2 \eta x^{2}\right)^{\frac{1}{2}}}{T}} \pm 1} d p \\
& =\frac{g}{\pi^{2} c^{3}\left(2 \eta x^{2}-1\right)\left(\eta x^{2}-1\right)} \\
& \times \begin{cases}{\left[-\operatorname{Li}_{3}\left(-e^{\frac{E_{m i n}}{T}}\right) T^{3}+E_{\min } \mathrm{Li}_{2}\left(-e^{\frac{E_{m i n}}{T}}\right) T^{2}\right.} & \\
\left.+\frac{1}{2} E_{\min }^{2} \ln \left(e^{\frac{E_{\min }}{T}}+1\right) T-\frac{1}{6} E_{\min }^{3}\right] & \text { Fermions } \\
{\left[\operatorname{Li}_{3}\left(e^{\frac{E_{\min }}{T}}\right) T^{3}-E_{\min } \mathrm{Li}_{2}\left(e^{\frac{E_{\min }}{T}}\right) T^{2}\right.} & \\
\left.-\frac{1}{2} E_{\min }^{2} \ln \left(-e^{\frac{E_{m i n}}{T}}+1\right) T+\frac{1}{6} E_{\text {min }}^{3}\right] & \text { Bosons }\end{cases}
\end{aligned}
$$

where, $E_{\min }=P_{\min } c\left(1-\eta x^{2}\right)$. Also the subscript "eff" means that the parameter contains the minimal momentum effects too. Note that in the case of $\eta=0$, we obtain the ordinary number density of massless parameters.

To simplify these relations we use the definition of polylogarithm function $\operatorname{Li}_{s}(z)$ as a power series in $z$, which is also a Dirichlet series in $s$, to find the following relation up to $\mathcal{O}\left(P_{\text {min }}^{4}\right)$

$$
\begin{aligned}
n_{e f f} \approx & \frac{g}{\pi^{2} c^{3}\left(2 \eta x^{2}-1\right)\left(\eta x^{2}-1\right)} \\
& \times\left\{\begin{array}{l}
{\left[\frac{3}{4} \zeta(3) T^{3}-\frac{1}{12} E_{\text {min }}^{3}+\frac{1}{32} \frac{\left.E_{\text {min }}^{4}\right]}{T} \quad\right. \text { Fermions }} \\
{\left[\zeta(3) T^{3}-\frac{1}{4} E_{\text {min }}^{2} T+\frac{1}{12} E_{\text {min }}^{3}-\frac{1}{96} \frac{E_{\text {min }}^{4}}{T}\right] \quad \text { Bosons. }}
\end{array}\right.
\end{aligned}
$$

The behavior of the re-scaled number density of massless and massive particles in terms of temperature (in the unit of Joule) with a minimal momentum for both Fermions and Bosons are shown in Fig. 1. To plot of the figures in this paper, we have adopted some sample values of the $\eta$. Note that, it is possible to have larger values of $\eta$. However, with larger values of $\eta$, the difference between the standard case and modified case becomes larger. Thus, it would be very hard to show both cases in the same plot. In this regard, we have adopted some suitable values of $\eta$, so we can show the difference between them in one plot. Also to generate all plots, we set $P_{\min }=\sqrt{\eta}$. The method of obtaining it is described in Ref. [5].

Due to the complexity and lengthful of the calculations of the massive thermodynamic quantities, we present them as an appendix and here we just perform the numerical analysis.

Then the energy density of such a massless system for Fermions and Bosons is given as follows 

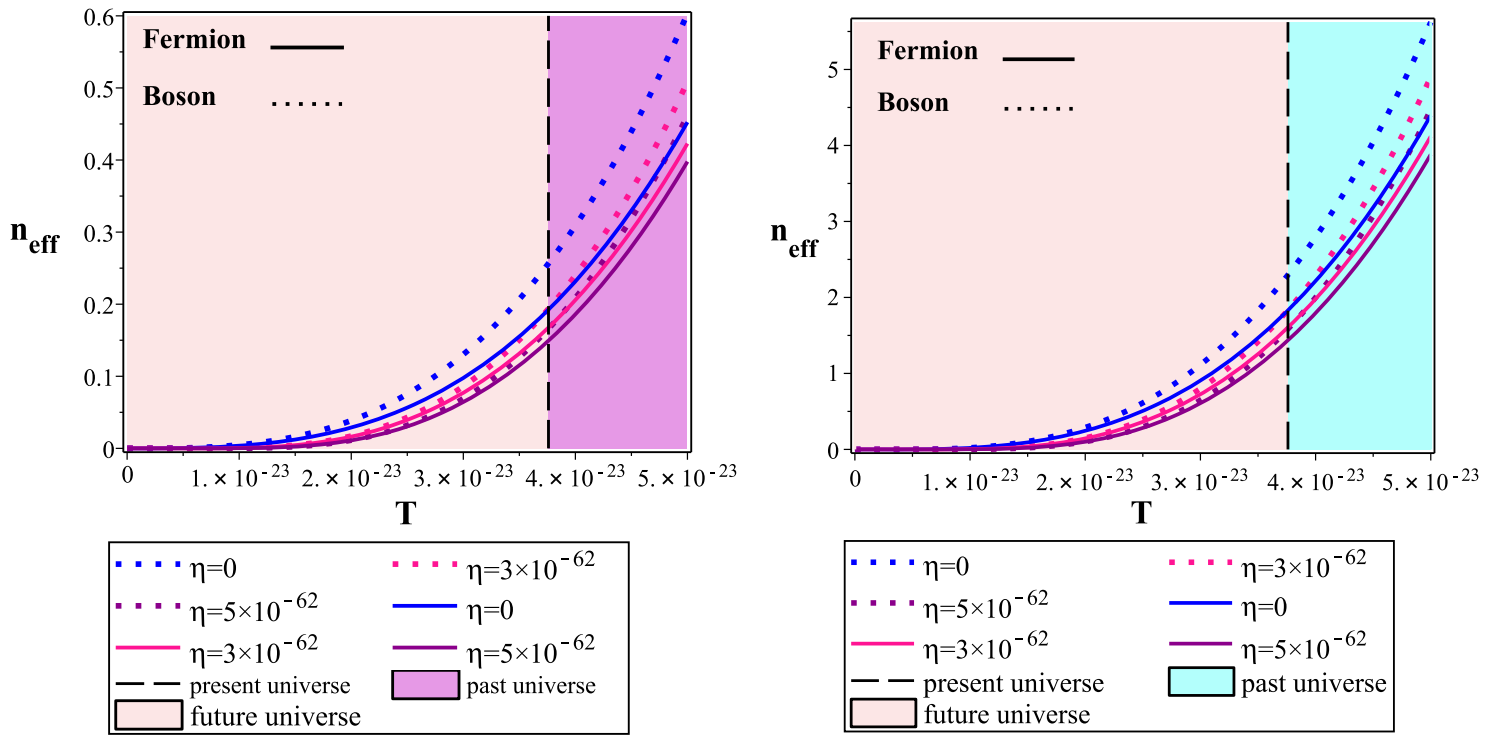

Fig. 1 The re-scaled number density of massless particles (left panel) and massive particles (right panel) versus temperature (in unit of Joule) with a minimal momentum for both Fermions and Bosons

$$
\begin{aligned}
& \rho_{e f f}=\frac{4 \pi g c\left(1-2 \eta x^{2}\right)^{\frac{1}{2}}}{(2 \pi)^{3}} \int_{P_{\min }}^{\infty} \frac{p^{3}}{e^{\frac{p c\left(1-2 \eta x^{2}\right)^{\frac{1}{2}}}{T}} \pm 1} d p=\frac{g}{\pi^{2} c^{3}\left(3 \eta x^{2}-1\right)}
\end{aligned}
$$

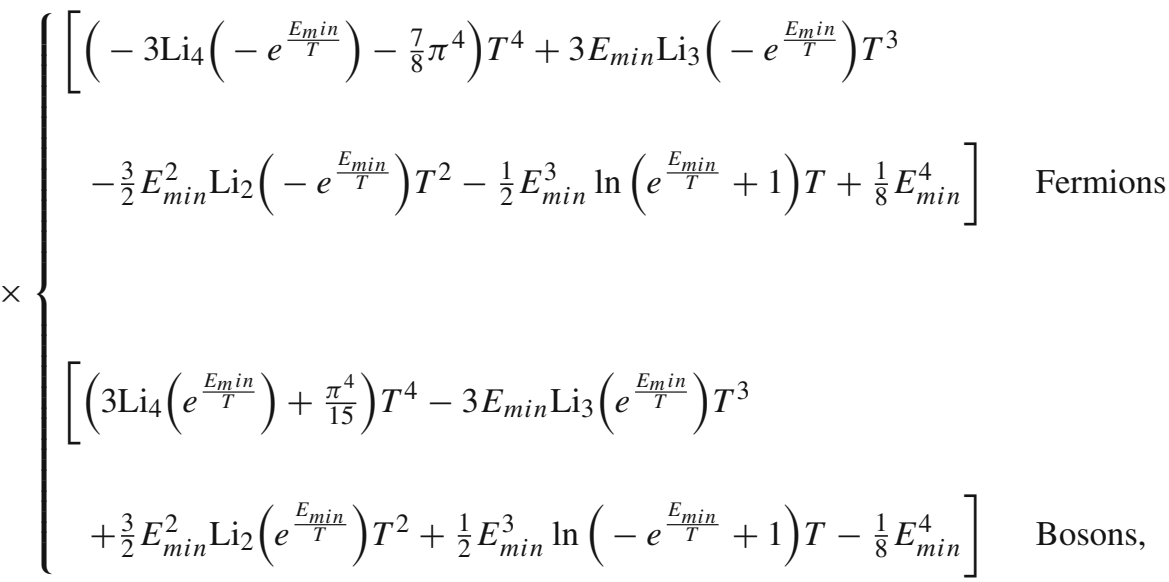

where up to $\mathcal{O}\left(P_{\text {min }}^{5}\right)$ gives

$$
\begin{aligned}
\rho_{e f f} \approx & \frac{g}{2 \pi^{2} c^{3}\left(3 \eta x^{2}-1\right)} \\
& \times \begin{cases}{\left[-\frac{7}{120} \pi^{4} T^{4}+\frac{1}{8} E_{m i n}^{4}\right]} & \text { Fermions } \\
{\left[-\frac{1}{15} \pi^{4} T^{4}+\frac{1}{3} E_{m i n}^{3} T-\frac{1}{8} E_{m i n}^{4}\right]} & \text { Bosons. }\end{cases}
\end{aligned}
$$

Figure 2 shows the behavior of the re-scaled energy density of massless and massive particles in terms of temperature with a minimal momentum for both Fermions and Bosons.

In the same manner, pressure of the massless species is calculated as follows 

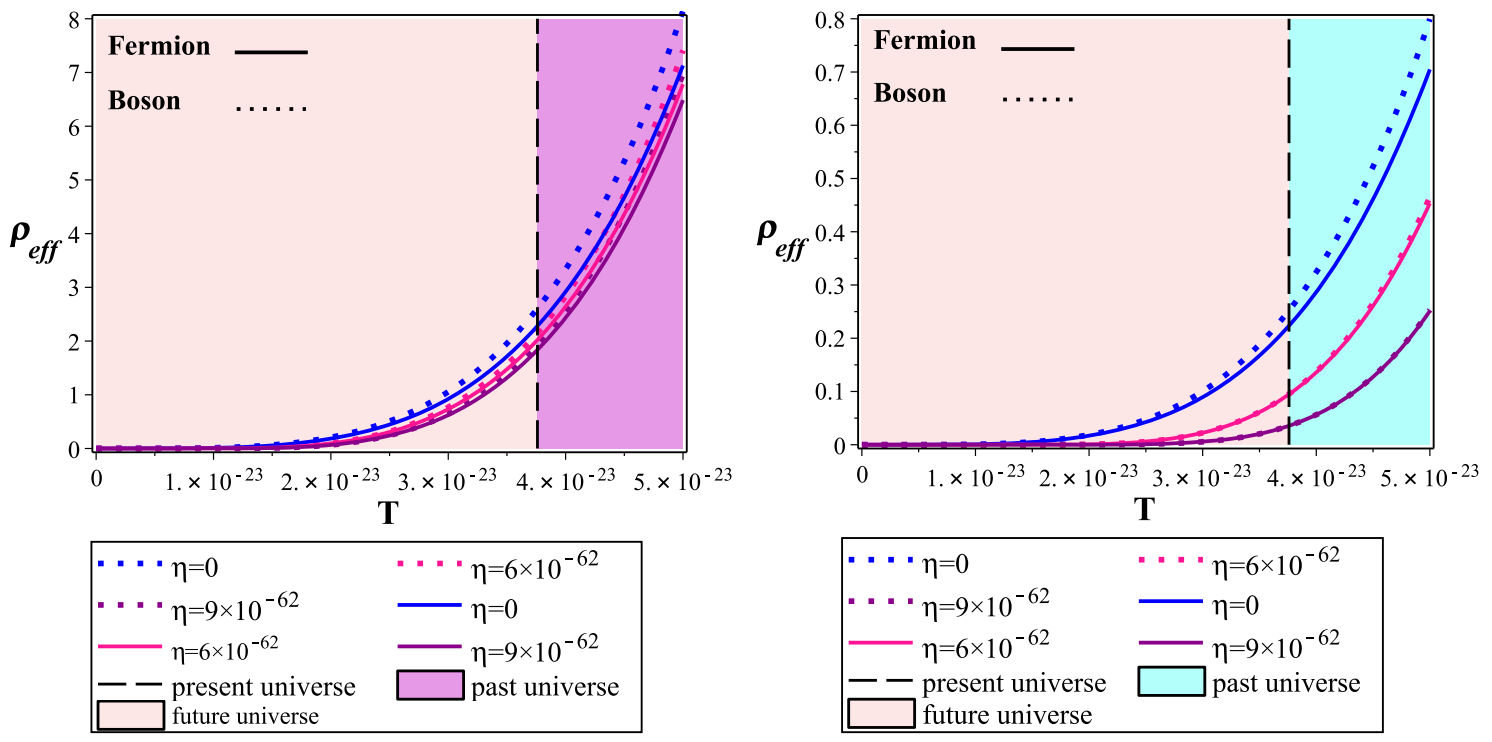

Fig. 2 The re-scaled energy density of massless particles (left panel) and massive particles (right panel) versus temperature (in Joule) with a minimal momentum for both Fermions and Bosons

$$
\begin{aligned}
& p_{\text {eff }}=\frac{\frac{4}{3} \pi g}{(2 \pi)^{3} c\left(1-2 \eta x^{2}\right)^{\frac{1}{2}}} \int_{P_{\min }}^{\infty} \frac{p^{3}}{e^{\frac{p c\left(1-2 \eta x^{2}\right)^{\frac{1}{2}}}{T}} \pm 1} d p=\frac{g}{\pi^{2} c^{5}\left(4 \eta x^{2}-1\right)\left(\eta x^{2}-1\right)}
\end{aligned}
$$

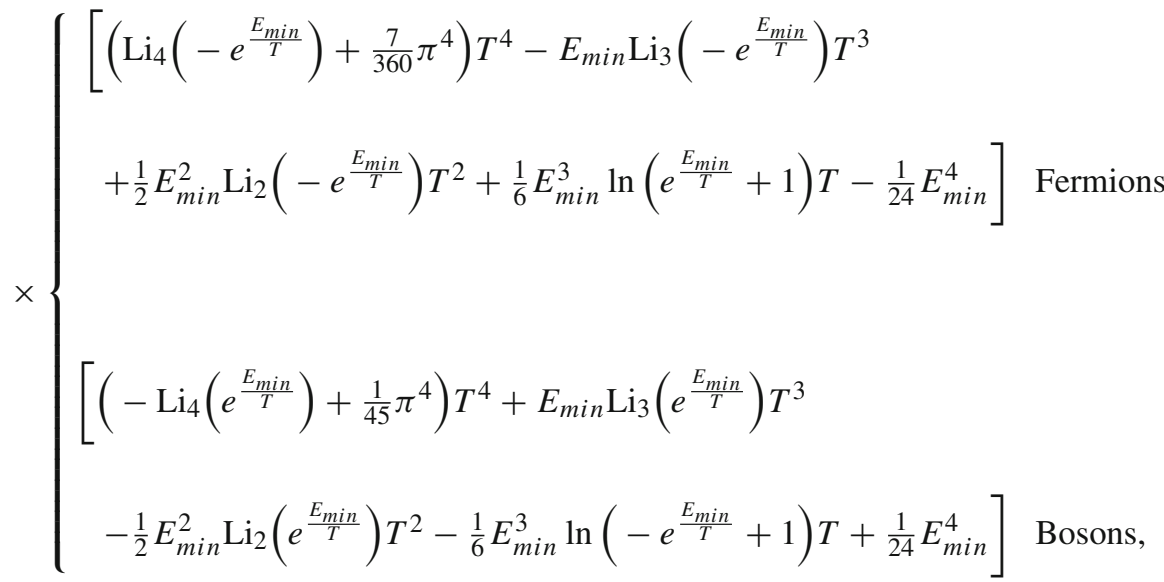

which can be approximated up to $\mathcal{O}\left(P_{\text {min }}^{5}\right)$ as

$$
\begin{aligned}
p_{e f f} \approx & \frac{g}{6 \pi^{2} c^{5}\left(2 \eta x^{2}-1\right)\left(\eta x^{2}-1\right)} \\
& \times \begin{cases}{\left[\frac{7}{120} \pi^{4} T^{4}-\frac{1}{8} E_{\text {min }}^{4}\right]} & \text { Fermions } \\
{\left[\frac{1}{15} \pi^{4} T^{4}-\frac{1}{3} E_{\text {min }}^{3} T+\frac{1}{8} E_{\text {min }}^{4}\right]} & \text { Bosons. }\end{cases}
\end{aligned}
$$

The behavior of the re-scaled pressure of massless and massive particles versus temperature with a minimal momentum for both Fermions and Bosons are shown in Fig. 3.

In thermal equilibrium at temperature $T$, the average particle energy is described by 

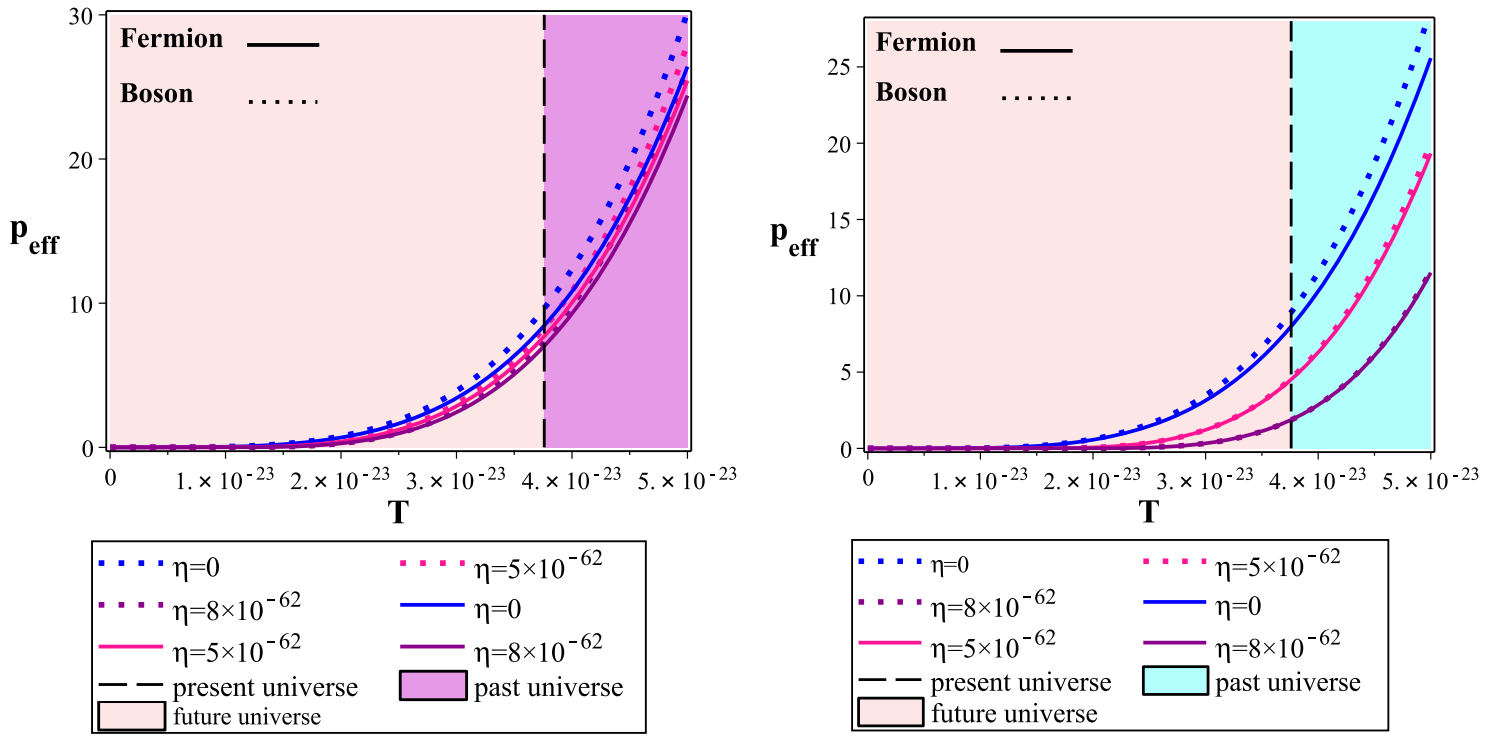

Fig. 3 The re-scaled pressure of massless particles (left panel) and massive particles (right panel) versus temperature (in unit of Joule) with a minimal momentum for both Fermions and Bosons

$$
\begin{aligned}
\langle E\rangle= & \frac{\rho}{n} \approx \frac{\left(\eta x^{2}-1\right)\left(2 \eta x^{2}-1\right)}{30\left(3 \eta x^{2}-1\right)} \\
& \times \begin{cases}\frac{1}{6}\left[-7 \frac{\pi^{4} T}{\zeta(3)}-\frac{7}{9} \frac{\pi^{4} E_{\min }^{3}}{\zeta(3)^{2} T^{2}}+\frac{1}{12} \frac{\left(\pi^{4}+60 \zeta(3)\right) E_{\min }^{3}}{\zeta(3)^{2} T^{2}}\right. \\
\left.-\frac{1}{96} \frac{\left(\pi^{4}(\zeta(3)+6)+180 \zeta(3)^{2}\right) E_{\min }^{4}}{\zeta(3)^{3} T^{3}}\right] & \text { Fermions } \\
{\left[-\frac{\pi^{4} T}{\zeta(3)}-\frac{1}{4} \frac{\pi^{4} E_{\min }^{2}}{\zeta(3)^{2} T^{2}}+\frac{1}{144} \frac{\left(7 \pi^{4}+360 \zeta(3)\right) E_{\min }^{4}}{\zeta(3)^{2} T^{3}}\right]} & \text { Bosons. }\end{cases}
\end{aligned}
$$

\subsection{Enthalpy and specific heat capacity}

Enthalpy is a parameter which plays a central role in most thermodynamics modeling of natural laws. This quantity is more often used to measure heats of reaction than internal energy. When the temperature of a system increases, the kinetic and potential energies of the particles in the system increase too, which means that the enthalpy of the system increases. This argument is true for both constant volume and constant pressure cases. So, we pay our attention to calculation of Enthalpy of the late time universe in the presence of a minimal measurable momentum. Given that the internal energy $U$ of the system is $\frac{3}{2} N k_{B} T$, the enthalpy of the system is given as follows

$H=U+p V$ that is,

$$
\begin{aligned}
& H=\frac{3}{2} N k_{B} T+\frac{g V}{\pi^{2} c^{5}\left(4 \eta x^{2}-1\right)\left(\eta x^{2}-1\right)}
\end{aligned}
$$

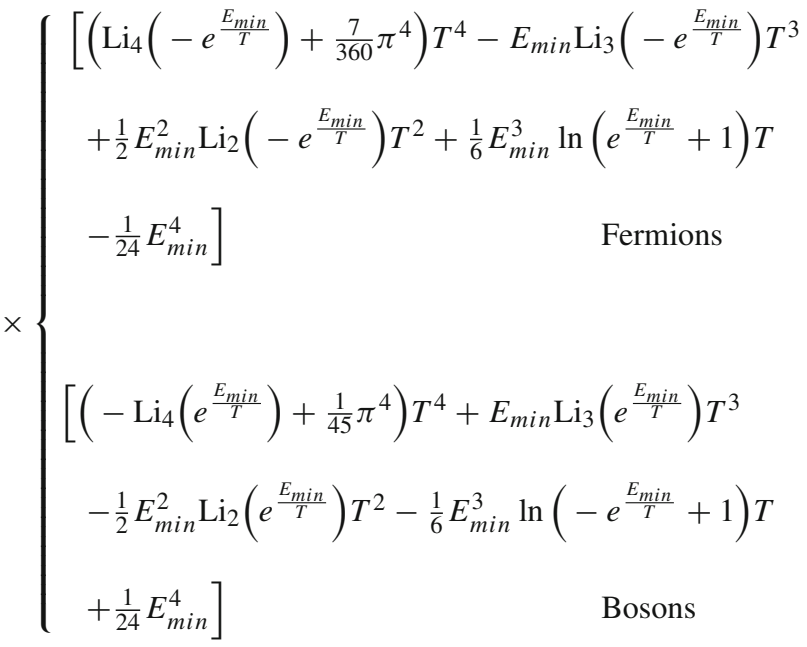

$$
\begin{aligned}
& \approx \frac{3}{2} N k_{B} T+\frac{g V}{6 \pi^{2} c^{5}\left(2 \eta x^{2}-1\right)\left(\eta x^{2}-1\right)} \\
& \times \begin{cases}{\left[\frac{7}{120} \pi^{4} T^{4}-\frac{1}{8} E_{\text {min }}^{4}\right]} & \text { Fermions } \\
{\left[\frac{1}{15} \pi^{4} T^{4}-\frac{1}{3} E_{\text {min }}^{3} T+\frac{1}{8} E_{\text {min }}^{4}\right]} & \text { Bosons. }\end{cases}
\end{aligned}
$$

The modified specific heat capacity relevant when the volume is kept constant is the temperature derivative of the modified internal energy as

$$
C_{V}=\left(\frac{\partial U}{\partial T}\right)_{V}=\frac{3}{2} N k_{B} .
$$



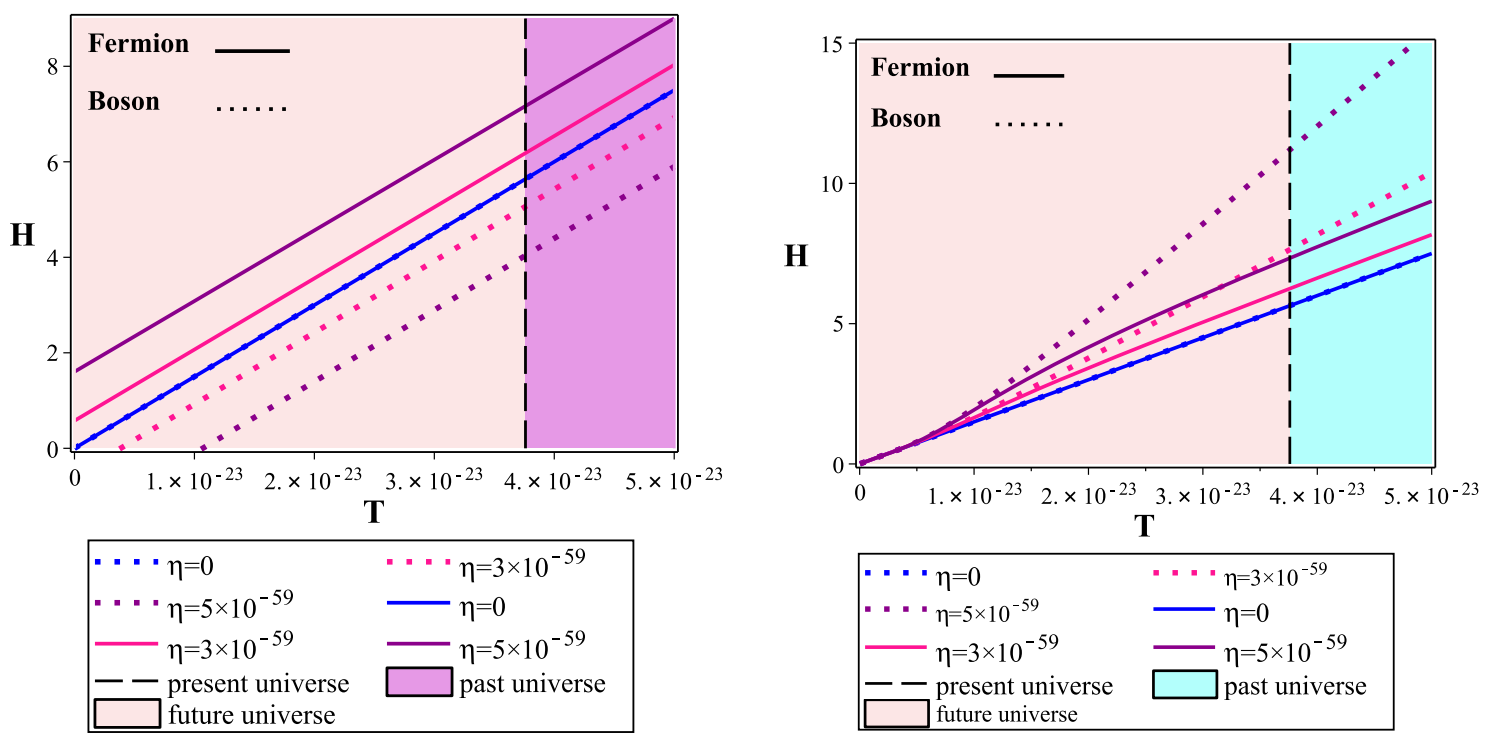

Fig. 4 The re-scaled Enthalpy of massless particles (left panel) and massive particles (right panel) versus temperature with a minimal momentum for both Fermions and Bosons

When the pressure of the system is kept constant, the modified specific heat capacity is described as

$$
C_{p}=\left(\frac{\partial H}{\partial T}\right)_{p}
$$

that is,

$$
\begin{aligned}
& C_{p}=\frac{3}{2} N k_{B}+\frac{4 g V}{\pi^{2} c^{5}\left(4 \eta x^{2}-1\right)\left(\eta x^{2}-1\right)}
\end{aligned}
$$

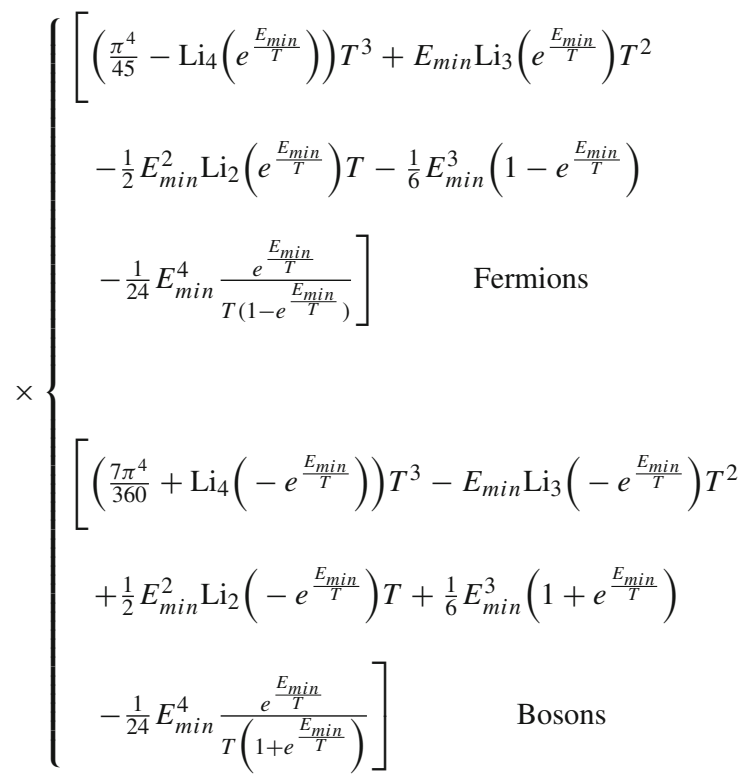

$$
\begin{aligned}
& \approx \frac{3}{2} N k_{B}+\frac{2 g V}{45 \pi^{2} c^{5}\left(4 \eta x^{2}-1\right)\left(\eta x^{2}-1\right)}
\end{aligned}
$$

$$
\times \begin{cases}{\left[7 \pi^{4} T^{5}-\frac{5}{4} E_{\text {min }}^{3} T^{2}+\frac{E_{\min }^{5}}{16}\right]} & \text { Fermions } \\ {\left[\frac{7}{8} \pi^{4} T^{5}-\frac{3}{16} E_{\text {min }}^{5}\right]} & \text { Bosons }\end{cases}
$$

where approximated up to $\mathcal{O}\left(P_{\text {min }}^{6}\right)$.

In Figs. 1, 2 and 3 qualitative behavior of the calculated quantities versus temperature are shown. In drawing these figures we have used the late time temperature of the cosmic background radiation which is about $2.725 \mathrm{~K}$ based on the PLANCK2018 observational data [17]. The temperature is stated in the unit of energy, that is, Joule. As is seen obviously in Fig. 1, in a given temperature at the late time universe and for a specific value of the parameter $\eta$, the density of states for massless Fermions is larger than the density of states of massless Bosons. However, note that in the absence of IR deformation, Bosons have higher density of states than Fermions in a given temperature. From Fig. 2, the energy density of massless Bosons for a given temperature is larger than the energy density of Fermions at that temperature with a fixed value of the IR deformation parameter $\eta$. The situation for pressure, as is shown in Fig. 3, is so that at a given temperature and in a fixed value of $\eta$, pressure of massless Bosons is larger than pressure of massless Fermions at that temperature. Another important point in this setup is the role of IR cutoff as a minimal measurable momentum, $P_{\min }$. Figure 1 shows that in the presence of IR cutoff, the density of states of both Fermions and Bosons are larger than the standard case with $\eta=0$. In other words, considering quantum gravitational effect as a natural IR cutoff at late time, modifies the density of states of species, so that this quantity gets enhanced rel- 

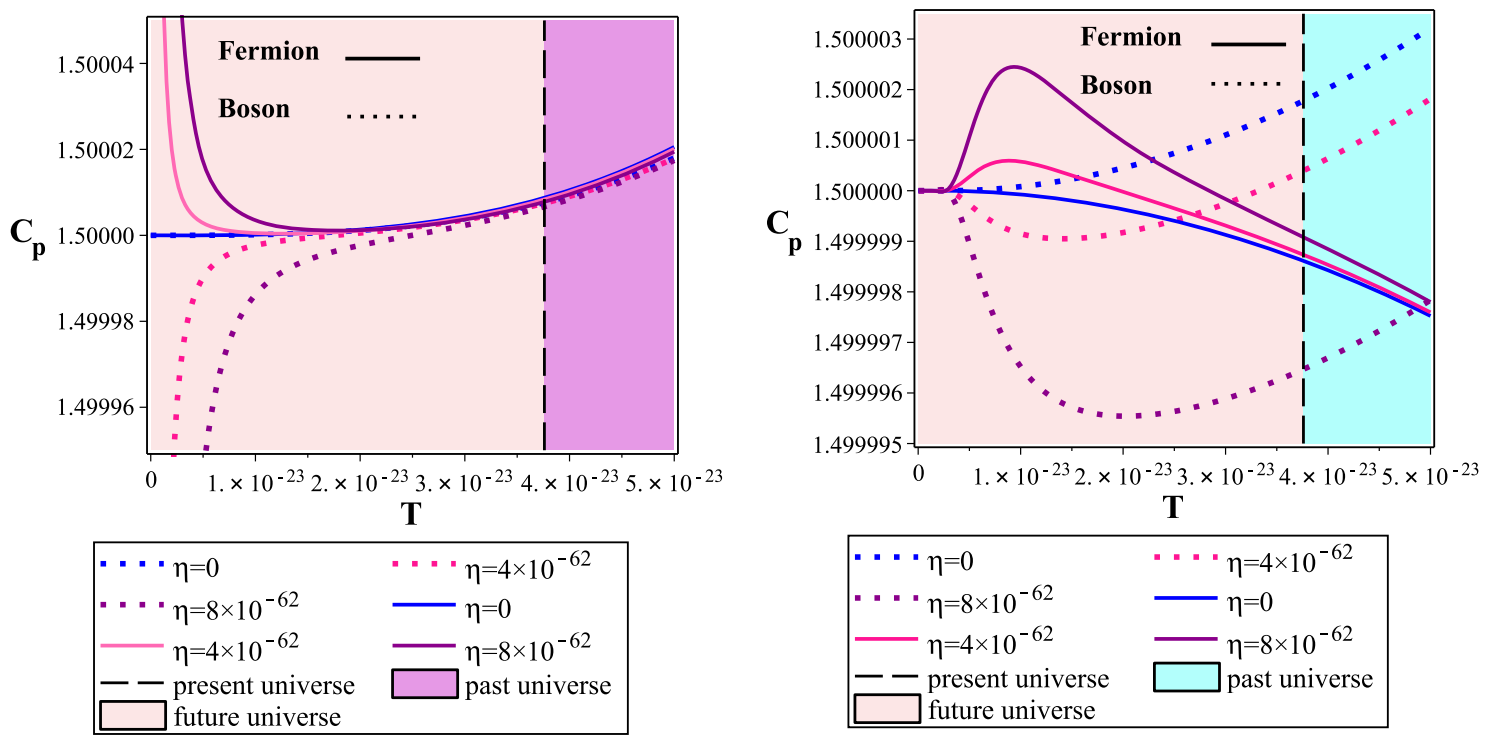

Fig. 5 The re-scaled specific heat capacity in constant pressure of massless particles (left panel) and massive particles (right panel) versus temperature with a minimal momentum for both Fermions and Bosons

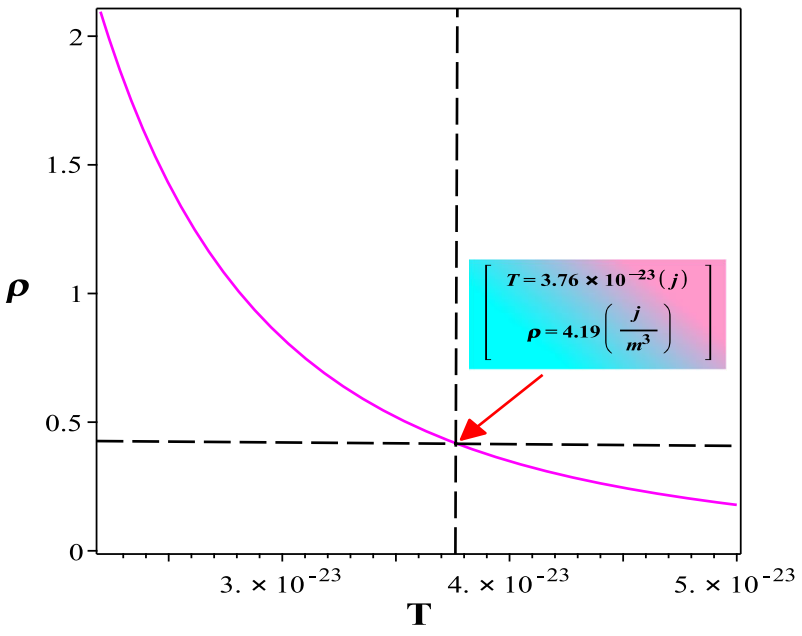

Fig. 6 The energy density $\left(\times 10^{-13}\right)$ versus temperature for massless particles (for instance, $\mathrm{CMB}$ photons) with a minimal momentum with $\eta=4.455 \times 10^{-46}$

ative to the standard case. The situation for energy density and pressure is more complicated and depends on the value of $\eta$. Figure 4 shows variation of the specific heat capacity in constant pressure versus the temperature for Bosons and Fermions in the presence of an IR cutoff.

The cosmic microwave background radiation today has the temperature 2.725 Kelvin which is equivalent to $3.76 \times$ $10^{-23}$ joules. As we know, the observational data shows that energy density of CMB is $4.19 \times 10^{-14}\left(\frac{j}{m^{3}}\right)$. Therefore, it seems that by considering the effects of IR cutoff in the late time universe with $\eta=4.455 \times 10^{-46}\left(\mathrm{~m}^{-2}\right)$, our model is observationally viable. In other words, one can justify the role of IR cutoff by comparing this observational value with the corresponding calculated value in the presence of IR cutoff. As Fig. 6 shows, there is good agreement between these two densities.

\subsection{Entropy}

Based on the second law of thermodynamics, the whole entropy of the universe never decreases, namely, it either stands constant or increases by cosmic evolution. Entropy generation in different processes in the universe evolution is negligible compared to the whole entropy of the universe. Hence, it is a viable assumption that expansion of the universe to be an adiabatic process, so that the entropy remains constant, that is, $d\left(s a^{3}\right)=0$. In this respect, it can be concluded that, there is a favorable relationship between $a(t)$ (scale factor) and temperature $T$ that keeps it constant as follows

$g_{s}(T) T^{3} a^{3}(t)=$ constant,

where $g_{s}$ is an effective number of entropy degrees of freedom.

To obtain entropy of the universe as a gaseous system containing Fermions and Bosons at the late time, we consider the following thermodynamic relation

$E=T S-p V+\sum_{i} \mu_{i} N_{i}$,

from which, we get the following result

$s=\frac{\rho+p-\sum_{i} \mu_{i} n_{i}}{T}$. 

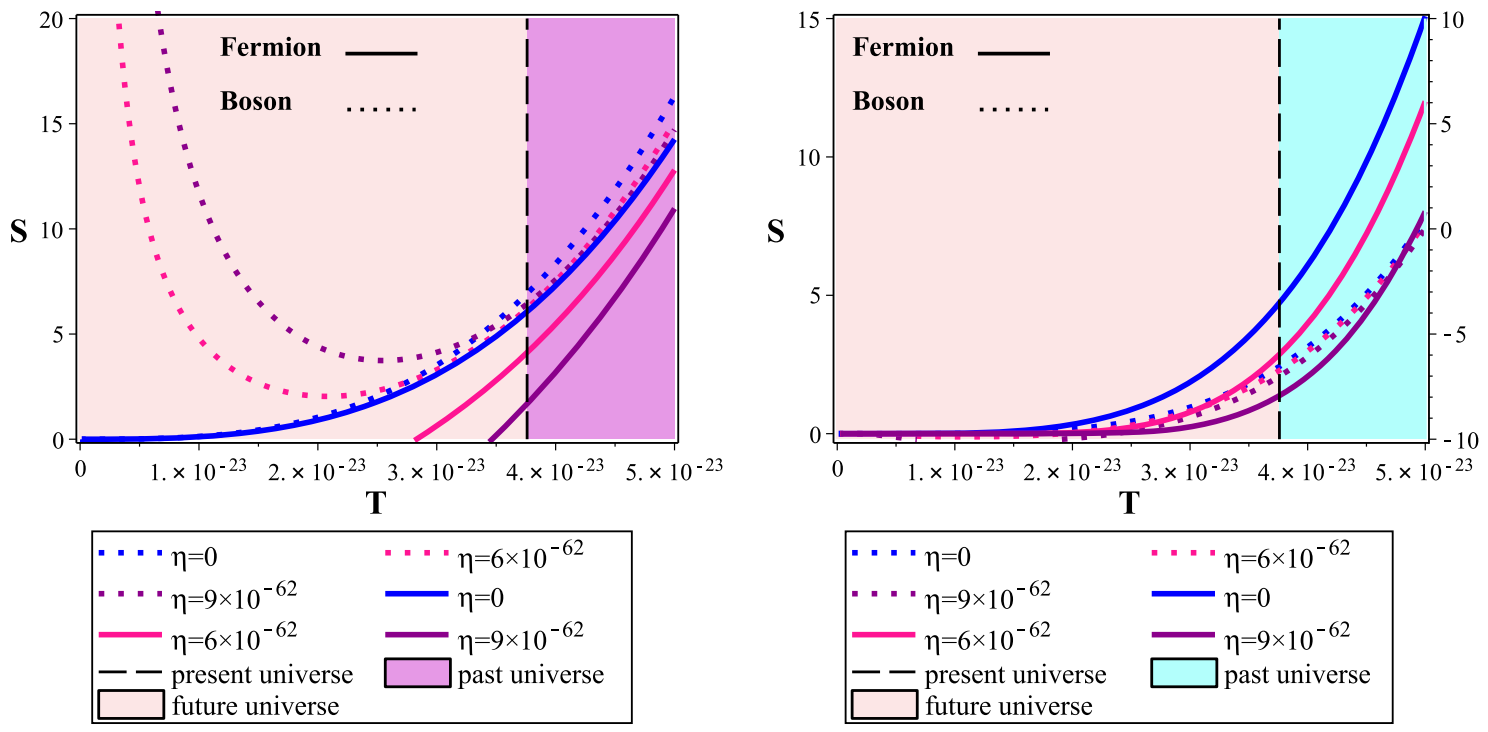

Fig. 7 The re-scaled entropy of massless particles (left panel) and massive particles (right panel) versus temperature with a minimal momentum for both Fermions and Bosons

For Fermions and Bosons sectors we find (with $\mu_{i}=0$ )

$$
\begin{aligned}
s= & \frac{\rho+p}{T}=\left(\frac{g \pi^{2}}{48 c^{5}}\right) \\
& \times\left(\frac{1+3 c^{2}-3 \eta x^{2}-15 c^{2} \eta x^{2}+12 c^{2} \eta^{2} x^{4}}{\left(3 \eta x^{2}-1\right)\left(\eta x^{2}-1\right)\left(4 \eta x^{2}-1\right)}\right) \\
& \times\left\{\begin{array}{l}
{\left[-\frac{7}{15} T^{3}+\frac{E_{\min }^{4}}{48 \pi^{4}} \frac{1}{T}\right] \quad \text { Fermions }} \\
{\left[-\frac{8}{15} T^{3}+\frac{8 E_{\min }^{3}}{3 \pi^{2}}-\frac{E_{\min }^{4}}{\pi^{2}} \frac{1}{T}\right] \text { Bosons }}
\end{array}\right.
\end{aligned}
$$

Figure 7 shows the behavior of the re-scaled entropy of the massless particles (left panel) and massive particles (right panel) versus temperature with a minimal momentum for both Fermions and Bosons.

According to our studies, the effects of the $\eta$ parameter are very small in the early universe. In other words, the difference between the standard model and the modified model is insignificant at the early universe. In fact, it was expected to be the case. This is because that, the effect of infrared cutoff, leading to the minimal momentum constraint, is related to the late time universe. Indeed, we didn't expect the quantities to change significantly in that period as well. In this article, we have focused on IR regime, for this purpose, we have drawn plots in the range of the late time universe temperature.

\section{Summary and conclusion}

Quantum gravitational effect as an infra-red cutoff can be encoded in the existence of a minimal observable momentum. In fact, in a curved background spacetime, momentum uncertainty of a test particle cannot be totally vanishing $[14,19]$. There is always a minimal uncertainty in momentum measurement due to the background curvature. This minimal uncertainty in momentum measurement nontrivially addresses the existence of an invariant, minimal momentum for a test particle. This phenomenological issue has a lot of outcomes in low energy physics. The present study is devoted to thermodynamics of late time universe in the presence of such an infra-red cutoff. In recent years it is believed that quantum gravitational effects may affect the large scale dynamics of the universe. Since quantum gravitational effect at low energy (or large distances) is reflected in the existence of an invariant minimal momentum (or maximal length, which can be considered as the size of the cosmological horizon today), it is important to see the effect of such a cutoff on the late time dynamics of the universe. We have considered a gaseous system as a model of the expanding universe. Both the massless and massive particles cases are considered in the study of universe's thermodynamics at late time. It is important to note that the notion of a gaseous system for ingredient of the late time universe is indeed sensible since we are working in a very low energy limit at the late time universe.

We have investigated the qualitative behavior of the re-scaled number density, re-scaled energy density and re-scaled pressure of massless and massive particles in terms of temperature 
with a minimal momentum for both Fermions and Bosons. We have shown for number density, in a given temperature at the late time universe and for a specific value of the parameter $\eta$, the density of states for massless Fermions is larger than the density of states of massless Bosons. However, note that in the absence of IR deformation, Bosons have higher density of states than Fermions in a given temperature.

Also, we have shown that the energy density of massless Bosons for a given temperature is larger than the energy density of Fermions at that temperature with a fixed value of the IR deformation parameter $\eta$. Furthermore, the qualification for pressure is so that at a given temperature and in a fixed value of $\eta$, pressure of massless Bosons is larger than pressure of massless Fermions at that temperature. Another important point in this setup is the role of IR cutoff as a minimal measurable momentum, $P_{\min }$. We have also concluded considering quantum gravitational effect as a natural IR cutoff at late time, modifies the density of states of species, so that this quantity gets enhanced relative to the standard case. We have also demonstrated that, there is significant agreement between the observational data and the corresponding parameter of the model in the presence of IR cutoff in massless case.

Data Availability Statement This manuscript has associated data in a data repository. [Authors' comment: There is no data to be deposited.]

Open Access This article is licensed under a Creative Commons Attribution 4.0 International License, which permits use, sharing, adaptation, distribution and reproduction in any medium or format, as long as you give appropriate credit to the original author(s) and the source, provide a link to the Creative Commons licence, and indicate if changes were made. The images or other third party material in this article are included in the article's Creative Commons licence, unless indicated otherwise in a credit line to the material. If material is not included in the article's Creative Commons licence and your intended use is not permitted by statutory regulation or exceeds the permitted use, you will need to obtain permission directly from the copyright holder. To view a copy of this licence, visit http://creativecomm ons.org/licenses/by/4.0/.

Funded by $\mathrm{SCOAP}^{3}$.

\section{Appendix}

Number density, energy density and pressure of massive case as follows

$$
\begin{aligned}
& n=\frac{4 \pi g}{(2 \pi)^{3}} \int_{P_{\min }}^{\infty} \frac{p^{2}}{e^{\frac{\left(m^{2} c^{4}+p^{2} c^{2}\left(1-2 \eta x^{2}\right)\right)^{\frac{1}{2}}}{T}} \pm 1} d p \\
& =\frac{g}{m^{2} \pi^{2} c^{3}\left(1-2 \eta x^{2}\right)^{\frac{3}{2}}}
\end{aligned}
$$

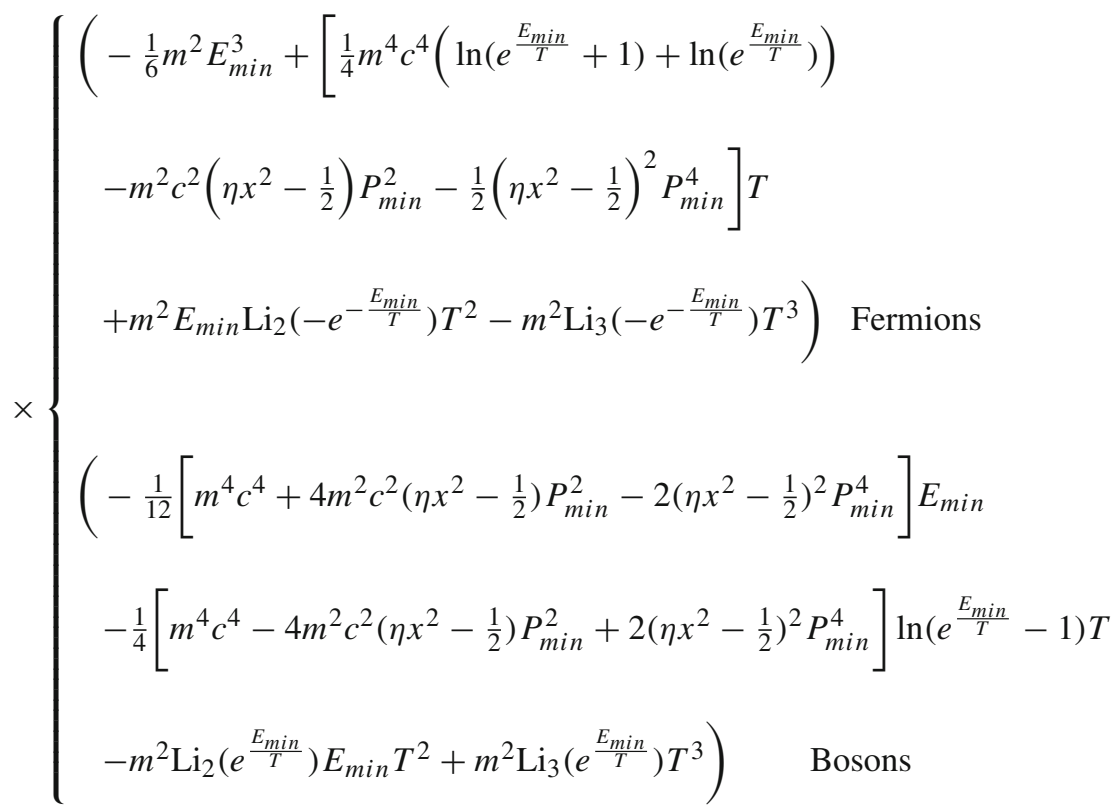


Eur. Phys. J. C (2020) 80:836

Page 11 of $12 \quad 836$

$$
\begin{aligned}
& \rho=\frac{4 \pi g c\left(1-2 \eta x^{2}\right)^{\frac{1}{2}}}{(2 \pi)^{3}} \int_{P_{\min }}^{\infty} \frac{p^{3}}{e^{\frac{\left(m^{2} c^{4}+p^{2} c^{2}\left(1-2 \eta x^{2}\right)\right)^{\frac{1}{2}}}{T}} \pm 1} d p \\
& =\frac{g}{\pi^{2} m^{6} c^{5}\left(2 \eta x^{2}-1\right)^{\frac{3}{2}}} \\
& \int\left(-\frac{7}{60} m^{3} E_{\text {min }}^{3}\left[m^{4} c^{4}+\frac{6}{7} m^{2} c^{2}\left(\eta x^{2}-\frac{1}{2}\right) P_{\text {min }}^{2}-\frac{3}{7}\left(\eta x^{2}-\frac{1}{2}\right)^{2} P_{\text {min }}^{4}\right]\right. \\
& +\frac{1}{4} m^{3} E_{\min }^{2} \ln \left(e^{\frac{E_{\min }}{T}}+1\right)\left[m^{4} c^{4}+2 m^{2} c^{2}\left(\eta x^{2}-\frac{1}{2}\right) P_{\min }^{2}-\left(\eta x^{2}-\frac{1}{2}\right)^{2} P_{\min }^{4}\right] T \\
& +2 m^{3}\left(\eta x^{2}-\frac{1}{2}\right) P_{\min }^{2}\left[\left(-\frac{1}{2} \eta x^{2}+\frac{1}{4}\right) P_{\min }^{2}+m^{2} c^{2}\right] E_{m i n} \mathrm{Li}_{2}\left(-e^{\frac{E_{\min }}{T}}\right) T^{2} \\
& \times 2 m^{3}\left[\frac{3}{2}\left(\eta x^{2}-\frac{1}{2}\right)^{2} P_{\min }^{4}-3\left(\eta x^{2}-\frac{1}{2}\right) m^{2} c^{2} P_{\min }^{2}+m^{4} c^{4}\right] \operatorname{Li}\left(-e^{\frac{E_{\min }}{T}}\right) T^{3} \\
& \left.-6 m^{5} E_{m i n} \operatorname{Li}_{4}\left(-e^{\frac{E_{\min }}{T}}\right) T^{4}+6 m^{5} \operatorname{Li}_{5}\left(-e^{\frac{E_{\min }}{T}}\right) T^{5}\right) \quad \text { Fermions } \\
& \left(\frac{7}{60} m^{3} E_{\min }^{3}\left[m^{4} c^{4}+\frac{6}{7} m^{2} c^{2}\left(\eta x^{2}-\frac{1}{2}\right) P_{\min }^{2}-\frac{3}{7}\left(\eta x^{2}-\frac{1}{2}\right)^{2} P_{\min }^{4}\right]\right. \\
& -\frac{1}{4} m^{3} E_{\text {min }}^{2} \ln \left(e^{\frac{E_{\min }}{T}}+1\right)\left[m^{4} c^{4}+2 m^{2} c^{2}\left(\eta x^{2}-\frac{1}{2}\right) P_{\min }^{2}-\left(\eta x^{2}-\frac{1}{2}\right)^{2} P_{\text {min }}^{4}\right] T \\
& -2 m^{3}\left(\eta x^{2}-\frac{1}{2}\right) P_{m i n}^{2}\left[\left(-\frac{1}{2} \eta x^{2}+\frac{1}{4}\right) P_{m i n}^{2}+m^{2} c^{2}\right] E_{m i n} \operatorname{Li}_{2}\left(e^{\frac{E_{m i n}}{T}}\right) T^{2} \\
& \times 2 m^{3}\left[\frac{3}{2}\left(\eta x^{2}-\frac{1}{2}\right)^{2} P_{\min }^{4}-3\left(\eta x^{2}-\frac{1}{2}\right) m^{2} c^{2} P_{\min }^{2}+m^{4} c^{4}\right] \operatorname{Li}\left(e^{\frac{E_{m i n}}{T}}\right) T^{3} \\
& \left.+6 m^{5} E_{m i n} \operatorname{Li}_{4}\left(e^{\frac{E_{\min }}{T}}\right) T^{4}-6 m^{5} \operatorname{Li}_{5}\left(e^{\frac{E_{\min }}{T}}\right) T^{5}\right) \quad \text { Bosons }
\end{aligned}
$$




$$
\begin{aligned}
& p=\frac{\frac{4}{3} \pi g}{(2 \pi)^{3} c\left(1-2 \eta x^{2}\right)^{\frac{1}{2}}} \int_{P_{\min }}^{\infty} \frac{p^{3}}{e^{\frac{\left(m^{2} c^{4}+p^{2} c^{2}\left(1-2 \eta x^{2}\right)\right)^{\frac{1}{2}}}{T}} \pm 1} d p \\
& =\frac{g}{\pi^{2} m^{2} c^{3}\left(2 \eta x^{2}-1\right)^{\frac{5}{2}}} \\
& \left\{\begin{array}{l}
\left(-\frac{1}{12} m^{3} E_{\text {min }}^{3}+\left[\frac { 1 } { 1 2 } m \left[m^{4} c^{4}-6 m^{2} c^{2}\left(\eta x^{2}-\frac{1}{2}\right) P_{m i n}^{2}\right.\right.\right. \\
\left.\left.+3\left(\eta x^{2}-\frac{1}{2}\right)^{2} P_{\text {min }}^{4}\right] \ln \left(e^{\frac{E_{\text {min }}}{T}}+1\right)+\frac{1}{6} \ln \left(e^{\frac{E_{\text {min }}}{T}}\right) m^{5} c^{4}\right] T
\end{array}\right. \\
& \left.+\frac{1}{2} m^{3} E_{m i n} \operatorname{Li}_{2}\left(-e^{\frac{E_{\min }}{T}}\right) T^{2}-\frac{1}{2} m^{3} \operatorname{Li}_{3}\left(-e^{\frac{E_{\min }}{T}}\right) T^{3}\right) \quad \text { Fermions } \\
& \times\{ \\
& \left(-\frac{1}{12} m\left[m^{4} c^{4}+2 m^{2} c^{2}\left(\eta x^{2}-\frac{1}{2}\right) P_{m i n}^{2}-\left(\eta x^{2}-\frac{1}{2}\right)^{2} P_{m i n}^{4}\right] E_{m i n}\right. \\
& -\frac{1}{12} m\left[m^{4} c^{4}-6 m^{2} c^{2}\left(\eta x^{2}-\frac{1}{2}\right) P_{\min }^{2}+3\left(\eta x^{2}-\frac{1}{2}\right)^{2} P_{\min }^{4}\right] \ln \left(e^{\frac{E_{\min }}{T}}-1\right) T \\
& \left.-\frac{1}{2} m^{3} E_{m i n} \operatorname{Li}_{2}\left(e^{\frac{E_{m i n}}{T}}\right) T^{2}+\frac{1}{2} m^{3} \operatorname{Li}_{3}\left(e^{\frac{E_{m i n}}{T}}\right) T^{3}\right) \quad \text { Bosons }
\end{aligned}
$$

\section{References}

1. G. Veneziano, Europhys. Lett. 2, 199 (1986)

2. D. Amati, M. Ciafabni, G. Veneziano, Phys. Lett. B 216, 41 (1989)

3. K. Konishi, G. Paffuti, P. Provero, Phys. Lett. B 234, 276 (1990)

4. A. Kempf, G. Mangano, R.B. Mann, Phys. Rev. D 52, 1108 (1995)

5. M. Roushan, K. Nozari, Adv. High Energy Phys. (2014) (Article ID 353192)

6. K. Nozari, M. Roushan, IJGMMP 13, 1650054 (2016)

7. E. Elizalde, S.D. Odintsov, I.L. Shapiro, Class. Quantum Gravity 11, 1607 (1994)

8. K. Nozari, B. Fazlpour, Chaos Solitons Fractals 32, 1-4 (2007)

9. D.N. Page, Int. J. Mod. Phys. D 19, 2271 (2010)

10. K. Nozari, P. Deghani, Phys. Lett. B 792, 101 (2019)
11. F.K. Anagnostopoulos, G. Kofinas, V. Zarikas, Int. J. Mod. Phys. D 28, 1944013 (2019). https://doi.org/10.1142/S0218271819440139

12. A.F. Ali, M. Mousa, Adv. High Energ. Phys. (2014). https://doi. org/10.1155/2014/629148

13. S. Gangopadhyay, A. Dutta, Adv. High Energ. Phys. (2018). https:// doi.org/10.1155/2018/7450607

14. H. Hinrichsen, A. Kempf, J. Math. Phys. 37, 2121 (1996)

15. S. Mignemi, Mod. Phys. Lett. A 25, 1697 (2010)

16. M. Roushan, K. Nozari, Eur. Phys. J. C 79, 212 (2019)

17. Planck Collaboration: Y. Akrami et al., Planck 2018 results. X. Constraints on inflation (2018). arXiv:1807.06211 [astro-ph.CO]

18. L. Perivolaropoulos, Phys. Rev. D 95, 103523 (2017)

19. M. Roushan, K. Nozari, IJGMMP 15, 1850136 (2018) 